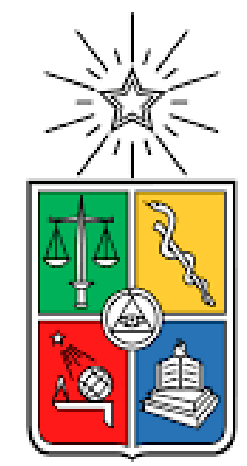

\title{
Desigualdad, política y lenguaje
}

\section{El rol del manejo del lenguaje en la transmisión intergeneracional de las habilidades políticas en jóvenes chilenos}

\author{
Francisco Javier Meneses Rivas \\ Juan Carlos Castillo (Profesor Guía)
}

Carrera de Sociología

Facultad de Ciencias Sociales

Universidad de Chile

28 de Agosto del 2020 
"Mi visión de la alfabetización va más allá del ba, be, bi, bo, bu; porque implica una comprensión crítica de la realidad social, política y económica en la que vive el alfabetizado"

Paulo Freire, 1992 


\section{Tabla de contenido}

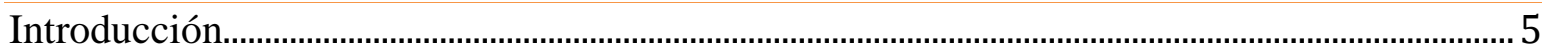

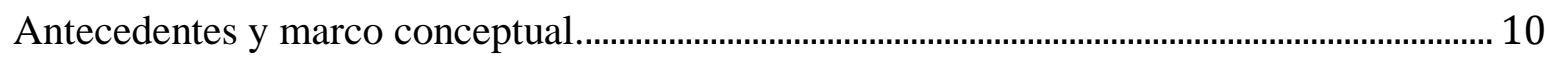

Politica, conocimiento cívico y desigualdad.......................................................................... 10

Manejo del lenguaje y Comprensión lectora ......................................................................... 16

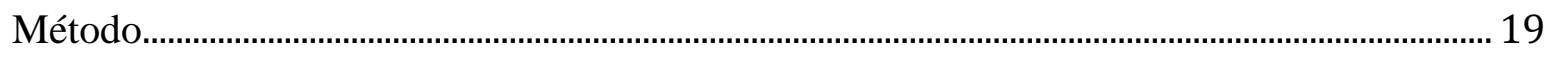

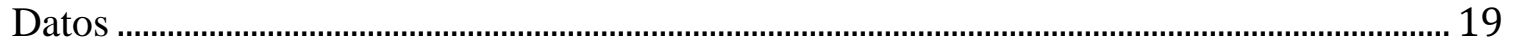

ICCS

SIMCE

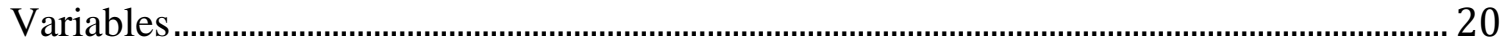

Prueba de conocimiento cívico y ciudadano.................................................................... 20

Prueba de comprensión lectora .................................................................................................... 22

Nivel socioeconómico del estudiante y del colegio................................................................. 23

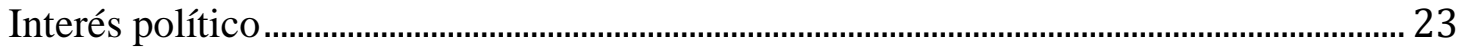

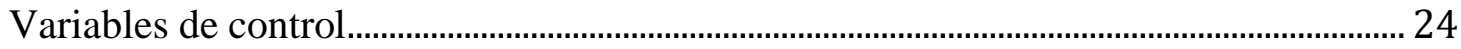

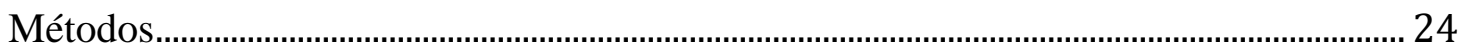

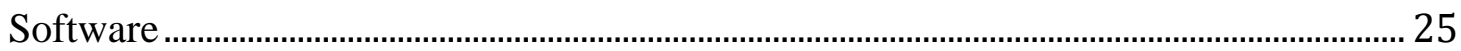

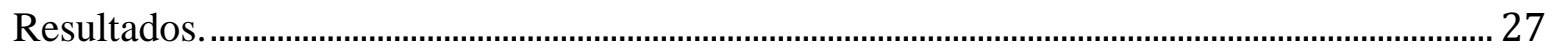

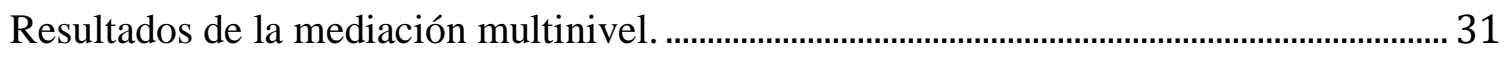

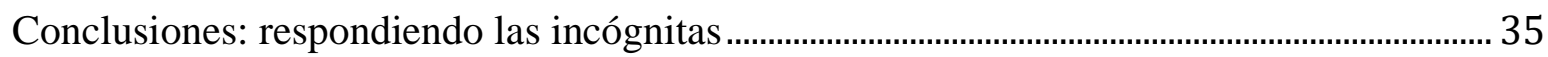

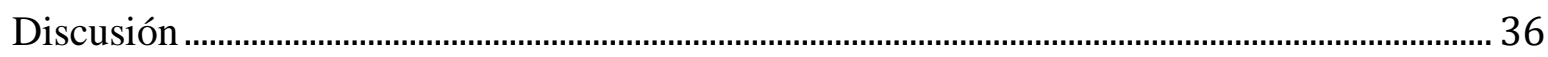

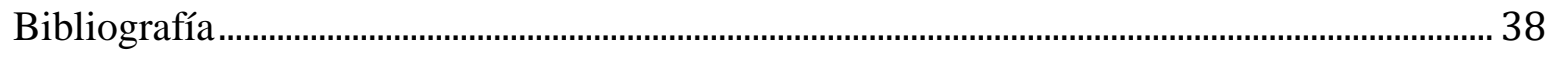


Resumen: Actualmente las democracias se encuentran frente a crisis de legitimidad que se relacionan, entre otras cosas, con la falta de representación de distintos grupos, como los sectores populares y los jóvenes. Al respecto, las investigaciones han señalado que no solo existen diferencias actitudinales hacia la política según grupo social, sino que también existen brechas en las habilidades políticas que se relacionan con la participación desigual. La asociación internacional para la evaluación del logro educativo (IEA) ha desarrollado el Estudio Internacional de Educación Cívica y Formación Ciudadana (ICCS) que se enfoca en estudiar las Habilidades y Conocimientos necesarios para la vida ciudadana en jóvenes estudiantes. El conocimiento cívico de la ICCS ha generado interés en distintos investigadores puesto que se asocia positivamente con un conjunto de actitudes democráticas como la participación y la tolerancia. Se ha demostrado que existe una relación entre nivel socioeconómico y conocimiento Cívico. Al explicar esta desigualdad algunos autores han señalado que en familias de alto capital cultural se genera un ambiente más ciudadano y académico, que implica una socialización en valores políticos y habilidades cognitivas. Para profundizar esta línea de investigación, se propone que parte de lo que explica la relación entre desigualdad social y conocimiento cívico es el manejo del lenguaje. Para evaluar esta hipótesis se trabajaron conjuntamente los datos de la ICCS y el SIMCE $(\mathrm{N}=3140)$. A partir de los análisis de mediación multinivel, se concluye que la transmisión intergeneracional de la desigualdad política se explica más por habilidades en el lenguaje que por la transmisión de intereses políticos. Además, se evidencia el potencial del lenguaje para disminuir la desigualdad de habilidades políticas, lo cual puede ser provechoso para la implementación del ramo de formación ciudadana.

Palabras clave: Conocimiento Cívico, Desigualdad política, Comprensión lectora, Interés político 


\section{Introducción}

Construir sociedades más democráticas es un objetivo internacional consagrado por la Organización de las Naciones Unidas tanto en la Declaración Universal de los Derechos Humanos como en el Pacto internacional de los Derechos Civiles y Políticos. Basándose en estos tratados internacionales, para que una sociedad sea efectivamente democrática son requisitos fundamentales una amplia participación de la ciudadanía y que la participación sea representativa, es decir, que no tenga sesgos socioeconómicos, raciales, de género, o de cualquier otra razón. Actualmente podemos decir que múltiples democracias poseen conflictos respecto a estas dos condiciones. En primer lugar, existe una tendencia a la disminución en la participación politica (Contreras y Navia, 2013; Díaz, 2017; Galston, 2007; Herrmann y Klaveren, 2016; Janmaat, 2013; Tezanos-Pinto, Cortés, y Concha, 2015). En segundo lugar, la participación politica es desigual, perjudicando la representación de la voz politica de sectores más pobres, de grupos racializados, de las mujeres y de los más jóvenes (Coffé y Lago, 2020; Desposato y Norrander, 2009; Hutchings y Valentino, 2004; Lijphart, 1997; Verba, 2003). Más aun, estas desigualdades políticas se transmiten de manera intergeneracional (Brady, Schlozman, y Verba, 2015). En el caso Chileno, tanto el Centro de Estudio de Conflicto y Cohesión Social (COES) como el Programa de las Naciones Unidas para el Desarrollo (PNUD) han señalado que existe una gran desigualdad en la participación politica, la cual sobrerrepresenta los grupos acomodados del país favoreciendo la reproducción de la desigualdad (Joignant, Bargsted, Somma, y Campos, 2017; Palet, Aguirre, y Chile, 2017).

Con el objetivo de fomentar valores democráticos en los ciudadanos y afrontar los desafíos de las democracias, cada vez más países, entre ellos chile, han incorporado en sus planes de estudio un mayor espacio para temáticas ciudadanas (Keer, 2015). La escuela es un lugar estratégico para fomentar valores, principios y conductas democráticas, pues en ella se realiza un proceso de socialización donde los estudiantes incorporan normas sociales necesarias para la vida en su comunidad (Durkheim, 2010). En torno a la socialización ciudadana de los jóvenes en las escuelas existen más de cuarenta años acumulados de investigaciones desde las ciencias sociales (Torney, 1979). Estas investigaciones han 
evidenciado reiteradamente que los estudiantes de distintos grupos socioeconómicos poseen distintas actitudes e intenciones de participación, lo cual es un obstáculo para la realización de la democracia (Castillo, Miranda, Bonhomme, Cox, y Bascopé, 2014; Ferráns y Sandoval-Hernández, 2017; Miranda, 2018; Schulz et al., 2010; Treviño, Béjares, Villalobos, y Naranjo, 2017).

Además de estudiar las actitudes y conductas políticas de los estudiantes, desde 1990, existe un esfuerzo por medir y estudiar las capacidades cognitivas de los jóvenes para su vida ciudadana (Torney-Purta, 2015). Este esfuerzo derivo en la prueba de conocimiento Cívico y Ciudadano la cual fue aplicada en tres encuestas internacionales de formación cívica. Según los creadores de la escala, esta busca medir tanto el conocimiento que poseen los estudiantes de sus sistemas políticos, como las habilidades cognitivas que poseen para reflexionar sobre situaciones concretas a partir de principios democráticos y su conocimiento político (Schulz y IEA, 2010). Existe contundente evidencia para señalar que un mayor conocimiento cívico se relaciona con mejores actitudes democráticas como la participación y la tolerancia (Galston, 2007; Miranda et al., 2018; Schulz y IEA, 2010). Una de las conclusiones más relevante de estos estudios es que existe una transmisión intergeneracional de la desigualdad politica, es decir, estudiantes hijos de padres con mejor situación económica, posen mejores conocimientos y habilidades para desenvolverse en la vida politica (Miranda, Castillo, y Sandoval-Hernandez, 2015; Schulz y IEA, 2010). Respecto a esta desigualdad, si bien se ha señalado que está estrechamente relacionada con aspectos de desigualdad cultural (i.e Educación de los padres y cantidad de libros en el hogar) y transmisión de actitudes, se ha dado poca relevancia a la transmisión de habilidades que ayudan a reproducir la desigualdad en la politica (Brady et al., 2015). En torno a las habilidades, la organización de Estados Americanos (OEA) señala que la carencia en alfabetización puede ser un obstáculo para la educación cívica en chile (Torney-Purta, 2015). Esta advertencia de la OEA, y algunas investigaciones que demuestran la dificultad de los estudiantes al leer la prueba por el lenguaje relativamente abstracto y complejo (Arensmeier, 2015; Zhang, Torney-Purta, y Mislevy, 2015), sugieren que el manejo del lenguaje puede jugar un rol en la transmisión de habilidades que favorecen la reproducción intergeneracional de la desigualdad politica 
En consideracion de lo señalado, la pregunta de esta investigación es:

\section{¿Cuál es el rol que juega el manejo del lenguaje en la transmisión intergeneracional de la desigualdad politica?}

Esta investigación busca evidenciar la importancia del manejo del lenguaje para explicar las desigualdades en habilidades políticas, frente a otras explicaciones basadas en la trasmisión de valores. Al explicar la reproducción intergeneracional de los conocimientos y habilidades políticas, las investigaciones recurren a dos líneas teóricas de la socialización, la actitudinal, centrada en la transmisión de valores e intereses, y la cognitiva, centrada en la transmisión de habilidades (Miranda, 2018). La propuesta de esta investigación plantea que la desigualdad de las habilidades cívico-ciudadanas se explica en una buena medida por desigualdades en habilidades, y más específicamente, por el manejo del lenguaje. Esta propuesta no solo se sustenta en el efecto de la cultura académica de los padres en las habilidades políticas, sino también en que la comprensión lectora requiere de las mismas destrezas cognitivas que el conocimiento cívico (Ej. Comprender, interpretar, analizar, evaluar, entre otras). Por ejemplo, para que un joven logre identificar una acción como antidemocrática, como alguien insultando a un inmigrante por su origen, no solo debe ser capaz de comprender que una persona le grita a otra, sino que debe interpretar cual es el sentido de esa acción y ser capaz de evaluar la relación entre dicho sentido y los principios democráticos, habilidades semejantes a las necesarias para la comprensión lectora.

En suma, se propone que el manejo del lenguaje posee una mejor capacidad mediadora que otras variables usualmente consideradas relevantes para explicar la desigualdad en el conocimiento Cívico, como puede ser el interés político (Diagrama 1). Si bien, efectivamente participar en un contexto de mayor capital cultural puede motivar un mayor interés político, el poseer este valor democrático no significa necesariamente que el estudiante posea la capacidad de responder satisfactoriamente la prueba de conocimiento y habilidades cívico-ciudadanas, ni poseer las habilidades necesarias para la vida ciudadana. Por el contrario, poseer un mayor manejo del lenguaje implica mayores capacidades 
cognitivas de diversos tipos que probablemente facilitan la comprensión del mundo político, la interiorización de conocimientos cívicos y la capacidad de evaluar diversas posturas. Si bien tanto intereses como habilidades son fundamentales para el desarrollo de un sujeto participativo, para explicar particularmente los conocimientos cívicos, es comprensible que las habilidades posean un mayor efecto.

\section{Diagrama $\mathrm{n}^{\circ} 1$ : Modelo teórico}

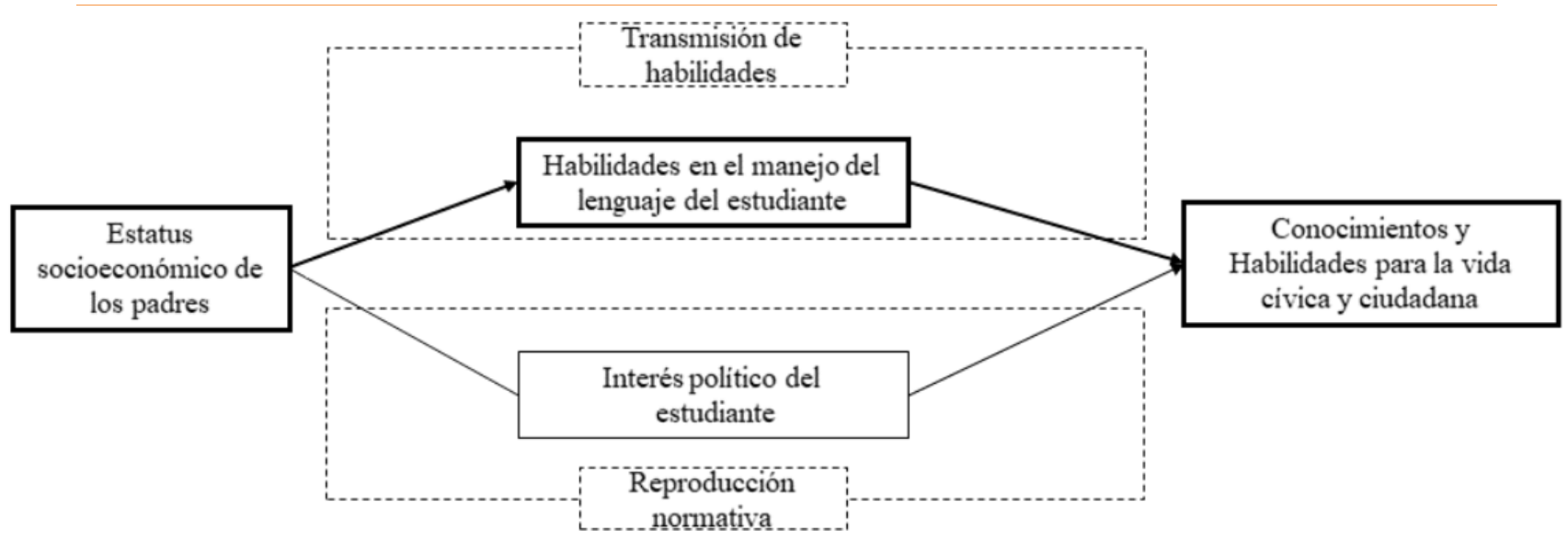

Fuente: Elaboración propia

Hipótesis: Las habilidades en el manejo del lenguaje explican en mayor medida la desigualdad social del conocimiento cívico que el interés político.

Evidenciar que el manejo del lenguaje es capaz de explicar en alguna medida la desigualdad social del conocimiento cívico es un aporte sustantivo a la teoría de la socialización politica. Que el lenguaje juegue un rol en las habilidades políticas daría cuenta de la importancia del manejo del lenguaje para la vida cívico-ciudadana. Esto es un aporte al modelo de recursos, pues permite profundizar en la comprensión del mecanismo causal de la reproducción social de la desigualdad politica, respecto a la transmisión de habilidades, como Brady et al. (2015) sugerían necesario.

Además, evidenciar el rol del lenguaje en el conocimiento cívico puede ser muy provechoso para las políticas públicas, ya que contar con información del contexto de aplicación del nuevo curso de formación ciudadana nos permite anticiparnos a sus posibles obstáculos. En primer lugar, podríamos suponer que la aplicación de un nuevo ramo de 
educación cívica, en el contexto de un socialmente desigual manejo del lenguaje, podría incrementar las brechas sociales en la materia, dado que los colegios de mayor nivel socioeconómico poseen un mayor manejo del lenguaje y por ello se encuentran en mejores condiciones para incorporar los conocimientos cívicos. En consideracion de esta posibilidad, seria provechoso para el objetivo del ramo, realizar planes de reforzamiento colectivo en lenguaje para aquellos colegios que poseen menores indicadores en las pruebas estandarizadas de comprensión lectora. En consideracion del mismo argumento y considerando las inequidades dentro de los estudiantes de un mismo curso, podría ser provechoso prestar asistencia psicopedagógica a estudiantes que posean un menor manejo del lenguaje, antes de que estos se enfrenten en el último ciclo de educación a la formación ciudadana. Para evaluar estas medidas, es necesario comprobar la capacidad que posee el lenguaje para disminuir la desigualdad de habilidades políticas.

A partir de las inquietudes y propuestas planteadas hasta ahora, se establecen los siguientes objetivos de Investigación.

- Objetivo general: Comprender el rol que cumple la desigualdad social del manejo del lenguaje en la influencia del origen socioeconómico sobre el conocimiento cívico y ciudadano.

- Objetivos específicos:

- Evaluar la relación entre comprensión lectora y conocimiento cívico incorporando factores influyentes señalados por la literatura.

- Contrastar si la desigualdad social del conocimiento cívico se explica por las diferencias de comprensión lectoras o por la transmisión de interés político.

Evaluar la capacidad que posee la comprensión lectora para resolver la desigualdad social del conocimiento cívico. 


\section{Antecedentes y marco conceptual.}

Para abordar la pregunta sobre la relación entre manejo del lenguaje y habilidades cívicas, es necesario desarrollar una conceptualización pertinente para ambos conceptos. Adicionalmente, desde una perspectiva cuantitativa, debe evaluarse la relación entre los conceptos definidos y los instrumentos que buscan medirlos, así como la evidencia que existe sobre los factores que afectan dichos constructos.

\section{Politica, conocimiento cívico y desigualdad.}

Antes de definir habilidades políticas, es necesario hacer una sucinta definición de lo político, en la cual se enmarca la concepción de habilidades políticas. Al definir politica desde los diccionarios es posible encontrar diferencias sustantivas en torno al rol del ciudadano en ellas. Por ejemplo, la politica es definida en el diccionario de la universidad de Oxford (2020) como "la ciencia que trata de la organización de las sociedades humanas o actividades de los que gobiernan o aspiran a gobernar", el uso dado al termino politica en este artículo es más acorde con una acepción propuesta por la Real Academia Española, a saber, "Actividad del ciudadano cuando interviene en los asuntos públicos con su opinión, con su voto, o de cualquier otro modo" (RAE, 2014). Esta última definición, es conveniente puesto que posee el sentido democrático y no tecnicista de la política, sentido que es coherente con los tratados internacionales de la ONU, como con distintas definiciones académicas de politica. Por ejemplo, desde la perspectiva de Arendt (2009), Lechner (1984) o Mouffe (1999), la politica es una actividad de realización humana, que se establece en base a la discusión y resolución de las diferencias que son propias en los humanos. Considerando lo anterior, las habilidades para la politica serian equivalente de capacidades que son necesarias para la discusión ciudadana y la participación en la resolución de las disputas del terreno público.

Actualmente las habilidades para la vida politica son un constructo muy estudiado desde distintas disciplinas como las ciencias políticas, la psicología, la sociología, entre otras. Existen varias formas de medir las habilidades políticas en adultos, que implican diferentes concepciones sobre el concepto, aunque todas suponen la posibilidad de capacidades 
diferenciadas para ejercer la participación ciudadana. Una manera común de medirlo es resumir el concepto a conocimiento político factual, es decir, conocimiento sobre quienes poseen actualmente cargos políticos de relevancia o conocimiento sobre las leyes y el derecho [Petričević y Stockemer (2020); @ vanerkelWhyDonWe2020]. Otro modo de medir las habilidades políticas es incorporando otras dimensiones, comúnmente cognitivas, que se relacionan con la capacidad de evaluar noticias, candidatos o realización de compromisos políticos (Duval y Pétry, 2018; Mondak, 2020). Desde el punto de vista de este artículo, es fundamental incorporar ambas dimensiones, por lo cual consideramos pertinente definir las habilidades cívicas como un conjunto de conocimientos facticos y la capacidad cognitiva de aplicar dichos conocimientos en distintas situaciones de la vida politica.

Dentro de los estudios de las habilidades políticas, enmarcados en los estudios de socialización escolar, nace la propuesta de medir dichas habilidades a partir de una prueba estandarizada. Esta, es la prueba de conocimiento y habilidades para la vida cívica y ciudadana, presente en el estudio internacional ICCS. Esta prueba, considera dos dimensiones en las habilidades ciudadanas, el Dominio de contenido, es decir el conocimiento factico de los principios del sistema democrático, y el Dominio cognitivo, comprendido como el conjunto de habilidades necesarias para la vida ciudadana, como la comprensión, el análisis y la evaluación (Schulz, Fraillon, Ainley, Losito, y Keer, 2011). Los conocimientos evaluados en esta prueba pueden apreciarse en la siguiente tabla. 


\section{Tabla $\mathrm{n}^{\circ} 1$ : Dominios de contenido del conocimiento cívico}

\begin{tabular}{|c|c|c|}
\hline Dominio & Descripciōn & Subdominios \\
\hline $\begin{array}{l}\text { Sociedad y } \\
\text { sistemas cívicos }\end{array}$ & $\begin{array}{l}\text { Este dominio se centra en los mecanismos formales e } \\
\text { informales y en las organizaciones que sustentan tanto } \\
\text { los contratos cívicos que tienen los ciudadanos con sus } \\
\text { sociedades, como el funcionamiento de dichas sociedades. }\end{array}$ & $\begin{array}{ll}\text { A. } & \text { Ciudadanos } \\
\text { B. Instituciones estatales } \\
\text { C. Instituciones civiles }\end{array}$ \\
\hline $\begin{array}{l}\text { Principios } \\
\text { cívicos }\end{array}$ & $\begin{array}{l}\text { Este dominio se centra en los fundamentos éticos } \\
\text { compartidos de las sociedades cívicas. El marco considera } \\
\text { el apoyo, protección y promoción de estos principios como } \\
\text { responsabilidades cívicas, y como motivación frecuente } \\
\text { para la participación cívica de individuos o grupos. }\end{array}$ & $\begin{array}{l}\text { A. Equidad } \\
\text { B. Libertad } \\
\text { C. Sentido de comunidad } \\
\text { D. Estado de derecho }\end{array}$ \\
\hline $\begin{array}{l}\text { Participación } \\
\text { cívica }\end{array}$ & $\begin{array}{l}\text { Se refiere a las manifestaciones de las acciones de los } \\
\text { individuos en sus comunidades. Puede operar a cualquier } \\
\text { nivel de la comunidad y en cualquier contexto de la misma } \\
\text { (incluyendo la escuela como el contexto más próximo de } \\
\text { los estudiantes). El nivel de participación puede variar entre } \\
\text { la conciencia que se manifiesta a través de un compromiso } \\
\text { hasta la influencia. }\end{array}$ & $\begin{array}{l}\text { A. Toma de decisiones } \\
\text { B. Influencias } \\
\text { C. Participación en la } \\
\text { comunidad }\end{array}$ \\
\hline $\begin{array}{l}\text { Identidades } \\
\text { cívicas }\end{array}$ & $\begin{array}{l}\text { Incluye las funciones cívicas de los individuos y la percepción } \\
\text { de dichas funciones. ICCS asume que los individuos influyen } \\
\text { en las relaciones que mantienen con sus familiares, amigos y } \\
\text { comunidades cívicas, al mismo tiempo que son influenciados } \\
\text { por dichas relaciones. Por lo tanto, la identidad cívica de } \\
\text { cada individuo depende expresamente de una serie de } \\
\text { interrelaciones cívicas y personales. Este marco sostiene } \\
\text { y asume que los individuos poseen múltiples identidades } \\
\text { articuladas, y no una única identidad cívica. Las comunidades } \\
\text { cívicas incluyen puntos de referencia en diferentes niveles } \\
\text { que van desde la familia y la comunidad local, hasta regiones } \\
\text { geográficas y la comunidad global. }\end{array}$ & $\begin{array}{l}\text { A. Autoimagen cívica } \\
\text { B. Conectividad cívica }\end{array}$ \\
\hline
\end{tabular}

Fuente: Mineduc (2016)

Cabe destacar, que esta prueba de conocimiento cívico ya ha pasado por tres olas internacionales, a partir de las cuales se ha refinado las características psicométricas de la medición. Esta prueba consta tanto de preguntas de selección múltiple como preguntas abiertas, a continuación, se presenta un ejemplo de pregunta en la cual se miden dominios cognitivos de razonamiento y análisis. Al respecto es importante destacar como esta pregunta requiere tanto de conocimientos políticos, como de habilidades propias del lenguaje como es la interpretación. Al respecto, Schulz et al. (2011), señalan que las preguntas de razonamiento y análisis requieren del uso del lenguaje para interpretar información, relatar, justificar, integrar, generalizar, evaluar, sugerir soluciones o predecir. 
Imagen $\mathrm{n}^{\circ} 1$ : Ejemplo de pregunta de la prueba de conocimiento cívico

\title{
İtem liberado 1
}

\begin{tabular}{|l|l|l|l|}
\hline Puntaje máximo & 1 & Clave respuesta & 3 \\
\hline Dominio de contenido & Principios civicos & & \\
\hline Subdominio de contenido & Equidad & Aspecto de contenido & No aplica (en adelante, n/a) \\
\hline Dominio cognitivo & Razonamiento y análisis \\
\hline
\end{tabular}

Abajo encontrarăs un autoadhesivo que puede comprarse por Internet.

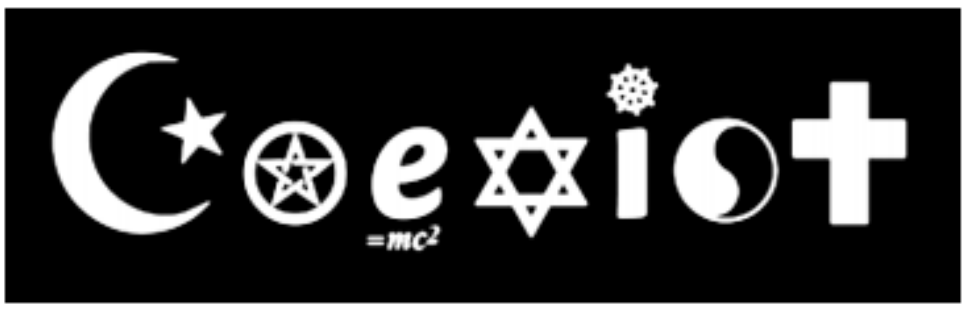

El autoadhesivo estă formado por simbolos que representan diferentes maneras de pensar sobre el mundo. Los símbolos han sido combinados para que se parezcan a la palabra inglesa "coexist" que significa "vivir juntos".

$\mathrm{Cl} 2 \mathrm{COM} 1$

P ¿Cuăl es el objetivo más probable de este autoadhesivo?

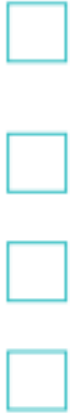

\begin{abstract}
Mostrar que las diferentes maneras de pensar son todas iguales.
Mostrar que las personas deberian reflexionar cuidadosamente sobre sus creencias.

Mostrar que las personas pueden aceptar a otros aunque tengan creencias diferentes.

Mostrar que las personas que tienen distintas maneras de pensar sobre el mundo nunca pueden vivir juntas en armonía.
\end{abstract}

Fuente: Mineduc (2016)

En torno a la evidencia levantada sobre el conocimiento cívico de estudiantes medido desde la propuesta de la ICCS, se han señalado fundamentalmente dos conclusiones: primero, el 
conocimiento cívico posee efectos positivos en valores y conductas democráticas, y segundo, el conocimiento cívico está influido por factores contextuales como la desigualdad social y la socialización escolar.

Debido a sus efectos positivos, el conocimiento cívico y ciudadano es actualmente promovido por diversos agentes a nivel académico, Estatal e internacional. Este conocimiento es sumamente relevante si se considera sus efectos positivos sobre la intención de participación (Miranda et al., 2015), en un contexto de apatía política y baja participación de estratos bajos y jóvenes (Contreras y Navia, 2013; Janmaat, 2013). Igualmente, en el contexto de los nuevos movimientos sociales que buscan reivindicar los derechos de distintos grupos tradicionalmente discriminados, el conocimiento cívico ha demostrado estar relacionado con el respeto a los derechos humanos de estos grupos (Caro y Schulz, 2012; Miranda et al., 2018). También, el tener más conocimiento cívico se relaciona con estar en desacuerdo con la corrupción y con la valoración positiva de la democracia como sistema representativo en contraposición a las dictaduras, lo cual, según Hastedt (2016), es fundamental en un contexto de resurgimiento de los gobiernos autoritarios. En suma, el conocimiento cívico puede ayudar a las personas a incorporar los principios democráticos de los derechos humanos.

Respecto a las variables que se relacionan con distintos niveles de conocimiento cívico, destacan la influencia del nivel socioeconómico y la influencia de las practicas democráticas en la escuela Las investigaciones actuales han propuesto que el conocimiento cívico es especialmente influido por variables de origen socioeconómico (ACE, 2017; Ferráns y Sandoval-Hernández, 2017; Schulz et al., 2011; Treviño et al., 2017), dando cuenta de lo que se denominará desde este punto, la "Desigualdad social del conocimiento cívico”. Este efecto del nivel socioeconómico, según la literatura, se debe menos al efecto de la ocupación de los padres que al efecto de variables culturales como la educación de los padres y el número de Libros, dando luces respecto al carácter cultural del fenómeno (Castillo et al., 2014). Además de los factores socioeconómicos y de transmisión cultural, otras investigaciones han enfatizado en el efecto producido por variables a nivel escuela como el nivel socioeconómico promedio de la escuela clima más abierto a la discusión y 
una cultura participativa a nivel escuela son propicios para el conocimiento cívico (Schulz et al., 2010). En suma, podemos ver que el conocimiento cívico es influido por distintas variables que se relacionan tanto con la socialización familiar como con la escolar, destacando las variables de reproducción cultural.

En consideracion de anterior, podemos decir que el conocimiento cívico es bastante explicado por el modelo de recursos, según el cual personas con mayores recursos poseen una mejor disposición a la politica (Miranda, 2018). Al explicar esta reproducción fundamentalmente cultural, la teoría de la socialización politica destaca dos dimensiones: la actitudinal y la cognitiva. La primera línea teórica destaca la trasmisión de valores e intereses políticos, suponiendo que convivir con padres con un mayor capital cultural conlleva tener conversaciones sobre temas políticos y sociales, que fomentan valores e intereses democráticos en los jóvenes, en contraste con la socialización apolítica de los jóvenes de estratos bajos (Gimpel, Lay, y Schuknecht, 2003; Wasburn y Adkins Covert, 2017). La segunda, destaca la transmisión de habilidades que son heredadas por la socialización familiar en una cultura académica con lectura temprana y acceso a libros (Evans, Kelley, Sikora, y Treiman, 2015; Park, 2008). Esta postura supone que esta cultura académica puede tener un efecto en la socialización politica (Boeve-de Pauw y Van Petegem, 2010; Duarte, Escario, y Sanagustín, 2017). Por su parte, la disponibilidad de libros en el hogar mejora el rendimiento académico e incrementa las capacidades intelectuales (Evans et al., 2015, 2015), mejorando las habilidades para la vida politica.

En resumen, las habilidades políticas o el conocimiento civico, son un conjunto de conocimientos y habilidades necesarias para la vida ciudadana, tales como memorización, la interpretación y la evaluación. A partir de la evidencia, se puede sostener que estas habilidades cívicas están muy relacionadas con la socialización politica, en la cual influye el origen social. Al explicar esta desigualdad social de las habilidades políticas, la teoría de la socialización recurre tanto a la transmisión de actitudes como de habilidades cognitivas. 


\section{Manejo del lenguaje y Comprensión lectora}

El lenguaje ha sido ampliamente conceptualizado a lo largo del pensamiento humano. Aristóteles (1988), concede al lenguaje un rol elemental cuando lo concibe como la herramienta propia del zoon politikon. Un rol semejante le otorga al lenguaje el filosofó precursor del romanticismo Herder, cuando plantea que esta es la habilidad que da coherencia a la actividad humana, siendo una herramienta que le permite comprender la realidad y reflexionar sobre ella, a partir de conceptualizarla. Desde ambos filósofos, y desde varios otros (ej. Echeverría, 2011; García, 2013), el lenguaje es aquello que nos permite relacionarnos como humanos, usar la razón y poder discutir sobre nuestros asuntos para coexistir. Ahora bien, aunque es cierto que el lenguaje es algo propio y por ende transversal en el genero humano, existe contundente evidencia para señalar que el manejo del lenguaje es diferenciado según grupos sociales.

Al respecto, el sociólogo contemporáneo de la línea de la reproducción cultural, Basil Berstein, realizo durante algunas décadas un amplio conjunto de experimentos e investigaciones para evaluar el uso del lenguaje en distintas clases sociales. En ellas, Bernstein (1985) concluye que los grupos económicamente acomodados y con mayores estudios, heredan a sus hijos un código sociolingüístico elaborado del lenguaje, que les permite hacer abstracciones y pensamientos que se separan de la situación contextual en la que se encuentran. Por el contrario, los jóvenes de los barrios obreros heredan gracias al proceso de socialización un código restringido el cual esta tendencialmente limitado a referencias contextuales y a situaciones vividas. Cabe destacar, como lo hace Bernstein (1988), que los grupos de clases medias y altas también poseen el Código restringido, pero poseen además el Código elaborado el cual utilizan en situaciones desafiantes. En suma, puede evidenciarse una desigualdad sociocultural en el manejo del lenguaje que genera capacidades diferenciadas de referenciar ideas fuera de lo vivido cotidianamente, diferencias las cuales explican según el autor la desigualdad de rendimiento académico entre clases sociales, ya que el conocimiento académico se sustenta en el Código elaborado, es decir, utiliza ideas fuera de la cotidianeidad vivida. En suma, podemos definir al lenguaje como la base de la reflexión, el cual puede presentar desarrollos diferenciados 
socioculturalmente, lo cual puede generar desigualdades en ámbitos que requieran de un lenguaje lejano a las vivencias cotidianas, como lo puede ser la academia o, desde nuestra propuesta, la politica.

Un indicador útil para medir la capacidad de los estudiantes de entender distintos discursos y ser capases de comprender ideas abstractas, así como analizarlas, interpretarlas y evaluarlas, es la comprensión lectora. La comprensión lectora implica la medición de la capacidad del estudiante, no solo de decodificar el texto, sino de comprender y analizar la relación entre las distintas partes de los textos, para posteriormente realizar ejercicios mentales más complejos como los son la síntesis, la interpretación la evaluación (ACE, 2018). Para trabajar con esta variable se utilizará la prueba SIMCE, la cual, justamente busca medir los logros de aprendizaje de los estudiantes chilenos.

Según Barahona U (2014) existe un consenso en que los factores asociados al desempeño académico en lenguaje pueden tener su origen en dos grandes ámbitos: en los determinantes personales y en los determinantes sociales. Así, una de las variables fundamentales para explicar el rendimiento en lenguaje es el nivel socioeconómico segun se plantea en el informe Coleman (Marqués, 2016). No obstante, no todo es reproducción social, pues como plantean Lara, Mizala, y Repetto (2010), las practicas docentes pueden tener un efecto positivo, por ejemplo, discutir la materia en clases es positivo para el rendimiento en comprensión lectora. Además de la participación en clases, el interés sobre la materia es un factor fundamental para su aprendizaje (Lozano, García-Cueto, y Gallo, 2000).

En suma, el manejo del lenguaje es una habilidad que permite la reflexión, la cual es fundamental para actividades que requieren de abstracción como lo es la académica y la politica. Este manejo, es adecuadamente medido por la prueba de comprensión lectora que evalúa habilidades como la comprensión, el análisis y la interpretación. Según la evidencia, el manejo del lenguaje es influido por variables socioeconómicas, por variables individuales; como el interés en la materia y por características de la escuela; como una educación participativa. 
Retomando nuestra hipótesis y robusteciéndola con las definiciones planteadas, podemos decir que el manejo del lenguaje, como herramienta del razonamiento, posee una influencia positiva en las habilidades políticas necesarias para la vida ciudadana, entre las cuales destacan la comprensión, la interpretación y la evaluación. Igualmente podemos decir que ambos conceptos son bien medidos por la prueba de conocimiento cívico y ciudadano, así como por las pruebas de lectura. Antes de entrar en el trabajo de datos de esta investigación (que será con las bases ICCS y Simce), se presenta una evidencia preliminar. A continuación, se puede observar un gráfico que expone la relación a nivel internacional, entre los promedios de los estudiantes por país de las pruebas de comprensión lectora de la prueba pisa y el conocimiento cívico de la ICCS.

\section{Gráfico $\mathrm{n}^{\circ} 1$ : Relación internacional entre conocimiento cívico y lenguaje}

\section{Relación entre conocimiento cívico (ICCS) y comprensión lectora (PISA) a nivel internacional}

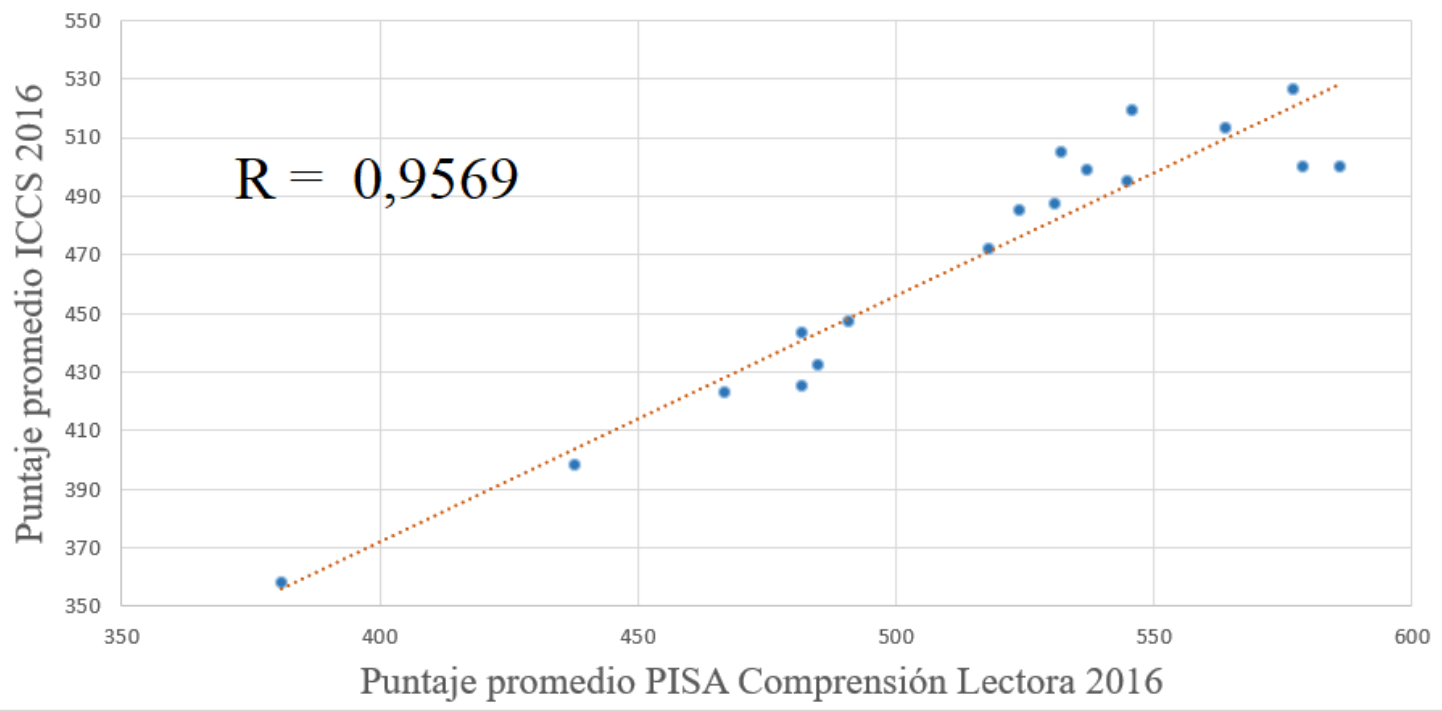

\section{Fuente: Elaboración propia}

Como puede apreciarse, a nivel internacional, existe una estrecha relación entre ambas variables. Según esta relación, países con mayores niveles de comprensión lectora poseen igualmente mayores niveles de conocimiento cívico y ciudadano. Como se expone en la imagen, existe una relación de alta intensidad entre ambas variables $(r=.95)$. No obstante, no hay que dejarse engañar por esta relación por dos razones. En primer lugar, esta relación 
no está controlada por ninguna variable. En segundo lugar, esta relación a nivel países, no nos permite afirmar que exista una relación entre comprensión lectora y conocimiento cívico a nivel individual o a nivel escuela. En consideracion de estas limitaciones, presentamos a continuación la propuesta metodológica para evaluar de un modo más certero esta hipótesis.

\section{Método}

\section{Datos}

\section{ICCS}

Para abordar la pregunta de investigación se trabajará con la base de datos de la encuesta internacional de conocimiento cívico (ICCS) en su tercera versión (2016). La ICCS es una encuesta enfocada en el estudio longitudinal del conocimiento cívico y las actitudes democráticas en estudiantes de octavo grado de 24 países. Dieciséis de esos países son de Europa, cinco de América Latina y tres de Asia, representados por 94.000 estudiantes y 37.000 profesores de 3.800 colegios (Schulz, Losito, Carstens, y Fraillon, 2016). Junto con una estrecha colaboración entre la IEA y los centros de estudios de cada país, los encargados de la elaboración de los datos fueron El Consejo Australiano para la Investigación Educativa (ACER) en Melbourne y el Laboratorio di Pedagogia Sperimentale (LPS) en la Universidad Roma Tre (IEA, 2016). La muestra de estudiantes posee un diseño estratificado de dos etapas, eligiendo escuelas al azar en un país y aulas al azar en una escuela, de tal modo que cada país tenga aproximadamente 150 escuelas y entre 3000 y 45000 estudiantes. La muestra de chile, con trabajo de campo en el 2015, incorporo colegios elegidos aleatoriamente, y representativos de distintas dependencias y regiones, tanto de sectores rurales como urbanos. De las escuelas seleccionadas aleatoriamente el $85 \%$ acepto participar, y dentro de las escuelas participantes, el $85 \%$ de los estudiantes acepto participar. En total, participaron 5.081 estudiantes de 178 establecimientos según señala la agencia de calidad de la educación (ACE, 2018). 


\section{SIMCE}

Adicionalmente se utilizaron los datos Simce del 2015, prueba censal que fue aplicada a los mismos estudiantes participantes del estudio de la ICCS, lo cual permite agregar estas bases de datos, logrando tener las variables de ambos estudios para cada estudiante de la muestra del ICCS. El Simce evalúa los logros de aprendizaje en las asignaturas de Lenguaje y Comunicación (Comprensión Lectura y Escritura); Matemática; Ciencias Naturales; Historia, Geografía y Ciencias Sociales e Inglés. En esta ocasión, hemos utilizado los datos de la prueba Simce de lenguaje y comunicación, más específicamente, de comprensión lectora. El estudio Simce, al igual que el ICCS, trabaja con el modelo de medición de Teoria de respuesta al ítem (ITR). "En particular [el Simce trabaja con] el modelo IRT de tres parámetros, [el cual] permite estimar la habilidad de un estudiante, basándose en la probabilidad de respuesta correcta según tres características propias de las preguntas: dificultad, discriminación y azar (ACE, 2018). Los procesos de aplicación de los instrumentos (pruebas y cuestionarios10) se llevan a cabo en función de instrucciones, manuales y protocolos de actuación que se basan en los criterios de estandarización internacional (AERA, 2011).

Respecto al tipo de preguntas, estas son de opción múltiple, pues este formato permite reportar información de la mayoría de los constructos a evaluar en forma efectiva y eficiente, asegurando validez, confiabilidad y objetividad del instrumento en su totalidad (Rupp y Böhme, 2008). En miras de este objetivo de la validez del instrumento, se realizan desde el año anterior a la aplicación pruebas piloto, las cuales son analizadas cualitativa y cuantitativamente para construir los indicadores más adecuados posibles. En suma, el Simce realiza una Logística de preparación que implica un gran gasto de recursos con el objetivo de reducir al máximo el error de medida.

\section{Variables}

\section{Prueba de conocimiento cívico y ciudadano}

La prueba de conocimiento cívico de la ICCS, tiene como objetivo evaluar los conocimientos necesarios para comprender y valorar la vida en sociedad y las formas de 
organización democrática, la capacidad de razonar acerca de las instituciones, eventos, acciones y procesos que se desarrollan en sus comunidades y la habilidad de desarrollar y justificar opiniones y visiones sobre estos elementos (Schulz y IEA, 2010). Los ítems de prueba requerían que los estudiantes aplicaran los procesos cognitivos al contenido cívico y de ciudadanía como se describe en el marco de evaluación del reporte técnico (Schulz et al., 2016). Más específicamente, la prueba de conocimiento Cívico y ciudadano mide:

- Dominios de contenido

- $\quad$ Sociedad y sistemas cívicos

- principios cívicos

- Participación cívica

- Identidades cívicas

- Dominios cognitivos

- conocimiento memorización

- Razonamiento y aplicación

Se utilizaron dos formatos de ítems: 79 de 88 ítems de la prueba son de opción múltiple con cuatro opciones de respuesta, como se puede ver en los ejemplos de los Anexos; los nueve ítems restantes fueron de respuesta construida para los cuales los estudiantes debían escribir entre una y tres oraciones. Para calcular los puntajes de cada estudiante, se parte de una media de 500 puntos, considerando una desviación estándar de 100 puntos, y según esta métrica se calculan 5 posibles resultados para cada estudiante. El que sean 5 resultados refleja el error y la incertidumbre propia de la medición de constructos complejos, al respecto se ha decidido trabajar con la primera estimación de puntaje. Según el reporte técnico (Schulz et al., 2016), se utilizó la teoría de respuesta al ítem. Para escalar los datos de prueba de ICCS 2016, los autores recurrieron al paquete de software de escalado ACER ConQuest, Versión 4. Posteriormente se realizaron diversas pruebas de validez, confiabilidad y equivalencia para comprobar que el conocimiento cívico es un constructo unidimensional, continuo y fiable. Además de las evaluaciones de fiabilidad, invarianza, equivalencia y validez realizadas por los centros de investigación designados por la IEA en el marco de la ICCS, otras agencias han evaluado la calidad de los cuestionarios. Así, por 
ejemplo, la agencia nacional de educación, evaluar el sesgo de deseabilidad social, concluyendo que la prueba no presenta problemas mayores de deseabilidad, y que estos se concentran en los aspectos de preguntas de actitudes y opiniones (MINEDUC, 2009)

\section{Prueba de comprensión lectora}

La comprensión lectora busca evaluar a los estudiantes en su capacidad de manejo del lenguaje y la comunicación. Específicamente, la prueba de lenguaje evalúa las siguientes actividades.

- Localizar información

- Identificar

- Discriminar

- Extraer

- Interpretar

- Inferir

- Interpretar lenguaje denotativo

- $\quad$ Reconocer relaciones causales

- Reflexionar

- Contrastar con conocimientos previos

- Evaluar críticamente aspecto de contenido y formato

Así, como puede apreciarse, para un buen puntaje en comprensión lectora, no solo se requiere la capacidad de decodificar el significado de las palabras, sino que se requiere un manejo del lenguaje a un nivel de razonamiento. Como ya se comentó, el proceso de elaboración y aplicación de la prueba tiene el objetivo de disminuir al mínimo variables externas al manejo del lenguaje que pudieran afectar la medición. Para evaluar las propiedades psicométricas de esta prueba, los encargados de la prueba Simce realizaron análisis en base a lineamientos internacionales (AERA, 2011). En base a los análisis realizados por el equipo técnico (ACE, 2018), podemos decir que la prueba de comprensión lectora es unidimensional (i.e El AFC indica un solo factor), posee independencia local (i.e estudiantes de un mismo rendimiento no presentan dificultades para determinada pregunta 
por factores externos) y motricidad creciente (i.e es un constructo continuo donde la probabilidad de responder correctamente un ítem aumenta progresivamente en estudiantes de mayor rendimiento).

\section{Nivel socioeconómico del estudiante y del colegio.}

Para medir el nivel socioeconómico de los estudiantes y de las escuelas se utilizará el Índice nacional de antecedentes socioeconómicos el cual es elaborado por el equipo de la e cuesta ICCS. Este índice es elaborado como un puntaje factorial que representa una variable latente relacionada con el mayor nivel ocupacional entre los padres, el mayor nivel educativo entre los padres y la cantidad de libros declarados en el hogar. Para evaluar el contexto socioeconómico del establecimiento educativo, se trabajará con el promedio por colegio del Índice nacional de antecedentes socioeconómicos. Adicionalmente, para realizar análisis más detallados, se utilizan de manera independiente las tres variables que componen dicho índice, para separar aspectos que refieren a desigualdades de capital cultural (educación de los padres y libros del hogar) del ítem que refiere a desigualdad económica (ocupación de los padres). Las variables educación de los padres y cantidad de libros serán dicotomizadas tanto para mejorar la parsimonia del modelo reduciendo los parámetros, como para evitar que existan categorías con muy pocos casos en los colegios, los cuales están altamente segregados socioeconómicamente.

\section{Interés político}

Para medir el interés político los estudiantes se utilizará la pregunta creada por la encuesta internacional ICCS. En este ítem se le pregunta al estudiante ¿Qué tan interesado está en temas políticos y sociales? (Muy interesado/ Bastante interesado / No muy interesado / No me interesa en absoluto). Para simplificar el análisis se ha recodificado esta pregunta de tal modo que las dos primeras alternativas se resumen en "Interesado en la politica" y las últimas dos en "No interesado en la politica". Cabe destacar que, para el análisis de mediación, se utilizó la variable original de 4 categorías centrada al promedio de la escuela. 


\section{Variables de control}

Como variables de control, será incluida la calidad de la discusión en el aula, ya que este es un indicador que ha sido consistentemente señalado como relevante por la evidencia, esta variable de nivel dos corresponde al promedio de los indicadores relacionados con las preguntas de clima en el aula.

\section{Métodos}

Respecto al método, este estudio trabajara desde un enfoque cuantitativo transversal. El uso de las herramientas cuantitativas es fundamental para despejar las dudas planteadas en este artículo, puesto que la cuantificación, como medición de lo social (Asún, 2006 en Canales, 2006), nos permitirá contrastar que variable posee una mejor capacidad mediadora de la reproducción de la desigualdad politica, la comprensión lectora o el interés político.

Se trabajará con técnicas de regresión multinivel, ya que estas son indispensables al estudiar muestras jerárquicas de colegios. Las regresiones multinivel, a diferencia de las regresiones normales, asumen que los estudiantes de un mismo establecimiento compartirán características debido al contexto común. Si se trabajara con regresiones lineales de un solo nivel, se rompería el supuesto de independencia de los casos en la muestra, ya que los casos están relacionados entre si al pertenecer a los mismos establecimientos. Esta metodología también nos permite evaluar el efecto de características de la escuela, como lo son el NSE promedio o la percepcion promedio de apertura a la discusión. Más específicamente, dentro del trabajo con regresiones multinivel, se trabajará con relaciones pendientes aleatorias, mediación multinivel e interacciones entre niveles.

Las pendientes aleatorias sirven para evaluar como varia la pendiente de una relación en distintos contextos, para este trabajo se calculará la variación de la pendiente de la relación entre conocimiento cívico y comprensión lectora. La medición multinivel permite evaluar una cadena causal considerando la estructura jerárquica de los datos. Para comprender esta metodología, es necesario entender que una medicación corresponde al fenómeno según el cual una variable "X", explica una variable "Y", por medio de "M", de tal modo que "X" genera "M" y M genera "Y" (Mathieu y Taylor, 2007). Al igual que en una mediación de 
un solo nivel, es requisito para comprobar la mediación multinivel, que " $\mathrm{X}$ " sea capaz de explicar tanto "M" como "Y", y que, además, M sea capaz de explicar "Y", controlando, en alguna medida el efecto de "X" (Baron y Kenny, 1986). En nuestro caso, queremos evaluar la capacidad de la comprensión lectora de mediar la relación entre NSE y conocimiento cívico, por ende, debemos evaluar la capacidad explicativa del NSE sobre la comprensión lectora y el conocimiento cívico (CC), y posteriormente, ver la capacidad del manejo del lenguaje de controlar el efecto del NSE sobre CC.

Considerando las recomendaciones de (Zhang, Zyphur, y Preacher, 2009) para cuando todas las variables del proceso de mediación se encuentran en el primer nivel, es fundamental, para evitar confusiones de los efectos producidas por la estructura jerárquica de la muestra, evaluar las relaciones en ambos niveles, es decir, un efecto de mediación intragrupo y otro entre grupos, para ello se deben realizar centrados en las medias de los grupos, según concluyeron los investigadores a partir de pruebas de simulación Montecarlo.

En suma, se evaluará la medicación de la comprensión lectora sobre la relación entre NSE y conocimiento Cívico, evaluando la relación a nivel dos y a nivel uno, incorporando centrados a la media del grupo. Se espera que el efecto de NSE sobre CC disminuya en buena medida al incluir el control de la comprensión lectora. además, se espera que la disminución del efecto por control sea mayor al incluir la variable comprensión lectora que interés político. En términos de interacciones entre niveles, evaluaremos la capacidad de la comprensión lectora del estudiante de moderar el efecto negativo sobre el conocimiento cívico que se debería de pertenecer a un establecimiento de bajo NSE promedio. Siguiendo las buenas prácticas para interacciones multinivel propuestas por (Aguinis, Gottfredson, y Culpepper, 2013), se centraron las variables según el promedio de la escuela, con la intención de despejar debidamente el componente individual de la varianza.

\section{Software}

El software de análisis estadístico utilizado fue R (versión 4.02) y la plataforma de edición

Github. Para los análisis multinivel recurrimos al paquete lme4, en su versión 1.1-23 (Bates, 2020). 
Además, siguiendo los lineamientos de la ciencia abierta, este trabajo está en un repositorio para facilitar tanto su acceso como su reproductibilidad. El lector de este seminario esta cordialmente invitado a visitar la página web del proyecto en la que se puede revisar tanto el articulo como los análisis. Igualmente, si el lector desea reproducir los análisis para verificar su veracidad, puede descartar el proyecto desde el repositorio de Github. Para facilitar la comprensión del orden de los archivos, estos se han ordenado según el esquema IPO, propuesto por Castillo (2020) 


\section{Resultados.}

En la tabla consecutiva se presentan 4 modelos multinivel los cuales son todos significativamente mejores que el anterior. El primer modelo incluye las variables de reproducción social, el segundo, incluye una variable de la escuela, el tercero agrega el interés político social del estudiante. El cuarto incluye la variable puntaje en la prueba Simce de lenguaje, y el quinto expone la aleatorización de la pendiente y la interacción de una variable nivel dos: el promedio del nivel socioeconómico.

\section{Tabla $n^{\circ} 2$ : Modelos multinivel}

\begin{tabular}{|c|c|c|c|c|c|}
\hline & \multicolumn{5}{|c|}{ Modelos para explicar nivel de Conocimiento Civico } \\
\hline & Modelo 1 & Modelo 2 & Modelo 3 & Modelo 4 & Modelo 5 \\
\hline \multirow[t]{2}{*}{ Intercepto } & $442.75^{88 *}$ & $293.88^{888}$ & $300.22^{* 8 *}$ & $125.40^{* 8 *}$ & $117.76^{888}$ \\
\hline & $(5.44)$ & $(33.64)$ & (33.61) & $(26.56)$ & $(25.92)$ \\
\hline \multirow[t]{2}{*}{ Más de 100 libros en el hogar } & $14.78^{* 8 *}$ & $14.48^{* * *}$ & $13.28^{* * 8}$ & $5.97^{*}$ & $5.94^{*}$ \\
\hline & $(2.96)$ & $(2.96)$ & $(2.96)$ & $(2.41)$ & $(2.41)$ \\
\hline \multirow[t]{2}{*}{ Estatus ocupacional de los padres } & $0.60^{* 8 *}$ & $0.60^{* * *}$ & $0.60^{* * *}$ & $0.36^{* * *}$ & $0.37^{* * *}$ \\
\hline & $(0.11)$ & $(0.11)$ & $(0.11)$ & $(0.09)$ & $(0.09)$ \\
\hline \multirow[t]{2}{*}{ Padres con educación universitaria o más } & $8.71^{*}$ & $8.98^{*}$ & $8.90^{*}$ & $7.96^{* *}$ & $7.72^{* *}$ \\
\hline & $(3.55)$ & (3.54) & (3.53) & $(2.86)$ & $(2.86)$ \\
\hline \multirow[t]{2}{*}{ Interes político de los padres } & $9.66^{* *}$ & $9.58^{* *}$ & 4.89 & 3.00 & 2.96 \\
\hline & $(2.97)$ & $(2.97)$ & $(3.08)$ & $(2.50)$ & $(2.50)$ \\
\hline \multirow[t]{2}{*}{ Media NSE de la escuela (M.NSE) } & $40.75^{* * *}$ & $37.46^{* * *}$ & $37.00^{* * *}$ & $18.24^{* * *}$ & $61.45^{* 3 *}$ \\
\hline & $(3.96)$ & $(3.85)$ & (3.84) & $(2.87)$ & $(11.23)$ \\
\hline \multirow[t]{2}{*}{ Cultura participativa de la escuela } & & $2.72^{* * *}$ & $2.60^{* * *}$ & $1.59^{* * *}$ & $1.63^{* * *}$ \\
\hline & & $(0.61)$ & $(0.61)$ & $(0.46)$ & $(0.45)$ \\
\hline \multirow[t]{2}{*}{ Interes político del estudiante } & & & $18.83^{* * *}$ & $13.40^{* 8 *}$ & $13.83^{8 * *}$ \\
\hline & & & $(3.45)$ & $(2.80)$ & $(2.80)$ \\
\hline \multirow[t]{2}{*}{ Comprensión lectora (C.Lec) } & & & & $1.01^{* * *}$ & $1.03^{* 8 *}$ \\
\hline & & & & $(0.03)$ & $(0.03)$ \\
\hline \multirow[t]{2}{*}{ Interacción: C.Lec*M.NSE } & & & & & $-0.15^{8 * 8}$ \\
\hline & & & & & $(0.04)$ \\
\hline $\mathrm{AIC}$ & 36221.01 & 36204.19 & 36176.54 & 34894.37 & 34880.68 \\
\hline $\mathrm{BIC}$ & 36269.43 & 36258.66 & 36237.06 & 34973.04 & 34965.41 \\
\hline Log Likelihood & -18102.51 & -18093.10 & -18078.27 & -17434.18 & -17426.34 \\
\hline Num. obs. & 3140 & 3140 & 3140 & 3140 & 3140 \\
\hline Num. groups: idschool & 152 & 152 & 152 & 152 & 152 \\
\hline Var: idschool (Intercept) & 824.00 & 683.87 & 685.93 & 4378.46 & 4371.31 \\
\hline Var: Residual & 5591.04 & 5591.74 & 5536.91 & 3648.77 & 3640.68 \\
\hline Var: idschool p.leng & & & & 0.03 & 0.03 \\
\hline Cov: idschool (Intercept) p.leng & & & & -12.22 & -12.08 \\
\hline
\end{tabular}

Fuente: Elaboración propia 
El primer modelo incluye aquellas variables que representan la reproducción social de la desigualdad política, y como puede verse, todas estas variables poseen un efecto significativo que implica cambios de puntajes que van aproximadamente desde 10 a 40 puntos. Así, poseer más de 100 libros en la casa, aumenta 14 puntos el conocimiento cívico, por su parte la variable ocupación posee un efecto considerable, en un rango de más de 80 valores, por cada unidad que aumenta el índice de estatus ocupacional el estudiante aumentó 0.6 puntos en la prueba de conocimiento cívico, así por ejemplo poseer 50 pts. más de estatus ocupacional implica aumentar 30 pts. en la prueba de conocimiento cívico. Respecto al efecto de la educación de los padres, este es ambivalente, puesto que, si bien es respaldado por la literatura, en vista de los controles aplicados parece solo tener un efecto significativo con un $95 \%$ de confianza, criterio demasiado laxo para una muestra de más de 3000 casos. En último lugar, considerando la variable de segundo nivel, promedio del nivel socioeconómico de los padres del colegio, podemos decir que existe un gran peso del contexto socioeconómico del colegio.

El segundo modelo incorpora una variable del contexto educativo, esta variable da cuenta de que tan de acuerdo están los estudiantes en general con que la participación en la escuela y la comunidad son beneficiosos para la comunidad. Como puede verse, por cada punto que aumenta este promedio mejora en 2 puntos el conocimiento cívico, lo cual reafirma la hipótesis de que un contexto de participación es útil para mejorar el conocimiento cívico de los estudiantes. Cabe destacar que el incorporar esta variable no logra controlar las variables de origen familiar, lo que nos da cuenta que las ventajas sociales del conocimiento cívico no pasan por estar en colegios con mejores climas democráticos.

El tercer modelo incorpora la variable interés político del estudiante. Como plantea la teoría, un estudiante que posee intereses sobre una materia posee una ventaja respecto a la misma, premisa que es comprobada en este caso. El que un estudiante posea interés en la política y los asuntos sociales se asocia con tener 19 puntos más en la prueba de conocimiento cívico. Resulta interesante que el efecto de poseer padres interesados por la política es completamente controlado por el efecto de que el estudiante tenga interés, lo que nos da cuenta de que el efecto de los padres pasa a través de los hijos. Este control nos 
permite decir que el efecto del nivel socioeconómico y las ventajas en términos de capital cultural no se explican completa, ni medianamente por qué las personas de mayores recursos tengan más intereses en la política, lo cual nos permite desde ya poner en duda las explicaciones que aluden a que el conocimiento cívico es transmitido como valores e intereses de generación en generación. Siendo más específicos, el efecto que más es controlados es el de tener libros en el hogar. Al parecer, el tener libros en la casa se relaciona con estudiantes interesados en la política, que obtuvieron mejores puntajes en esta prueba.

El cuarto modelo es el modelo fundamental de este trabajo. En este modelo las demás variables son controladas por el efecto de la comprensión lectora medida en la prueba simce de lenguaje. Como puede verse cada punto que aumenta alguien en la prueba simce de lenguaje, aumenta 1 punto en la prueba de conocimiento cívico, lo cual es una relación bastante estrecha considerando los rangos de ambas pruebas (hasta 350 pts). Lo más interesante de incorporar esta variable es lo que ocurre con las demás. Como puede verse el efecto de que existan más de 100 libros en el hogar, prácticamente desaparece, pasando de un efecto de 13 a 6 puntos, y perdiendo la significación al 99\%. Por su parte, respecto a la variable de estatus ocupacional de los padres y al nivel socioeconómico promedio del colegio, es bastante sugerente el hecho de que ambos efectos disminuyen casi a la mitad. En vista de estos controles, podemos decir que el efecto de que los padres posean libros en la casa es realmente producido por la influencia que eso ejerce en la comprensión lectora de los estudiantes. Igualmente, podemos decir que casi la mitad del efecto del nivel socioeconómico de los padres en el conocimiento cívico se debe a la influencia de estas posiciones en el buen manejo del lenguaje.

El quinto modelo es un poco más complejo. Primero incorpora la aleatorización de la pendiente del efecto de la comprensión lectora sobre el conocimiento cívico, permitiendo que esta varíe según contextos. Luego, intentamos explicar dicha variación de la pendiente en función del nivel socioeconómico del colegio, para ver si el efecto del lenguaje sobre el conocimiento cívico difiere en contextos socioeconómicos distintos. Como puede verse en la tabla, el efecto es significativo, y mientras mayor es el nivel socioeconómico menos 
importante es la comprensión lectora. Para graficar esta interacción, invertimos el sentido de esta. De este modo, se presenta a continuación la capacidad del lenguaje de moderar el efecto de la desigualdad producida por el NSE promedio del colegio.

\section{Gráfico $\mathrm{n}^{\circ}$ 2: Interacción multinivel}

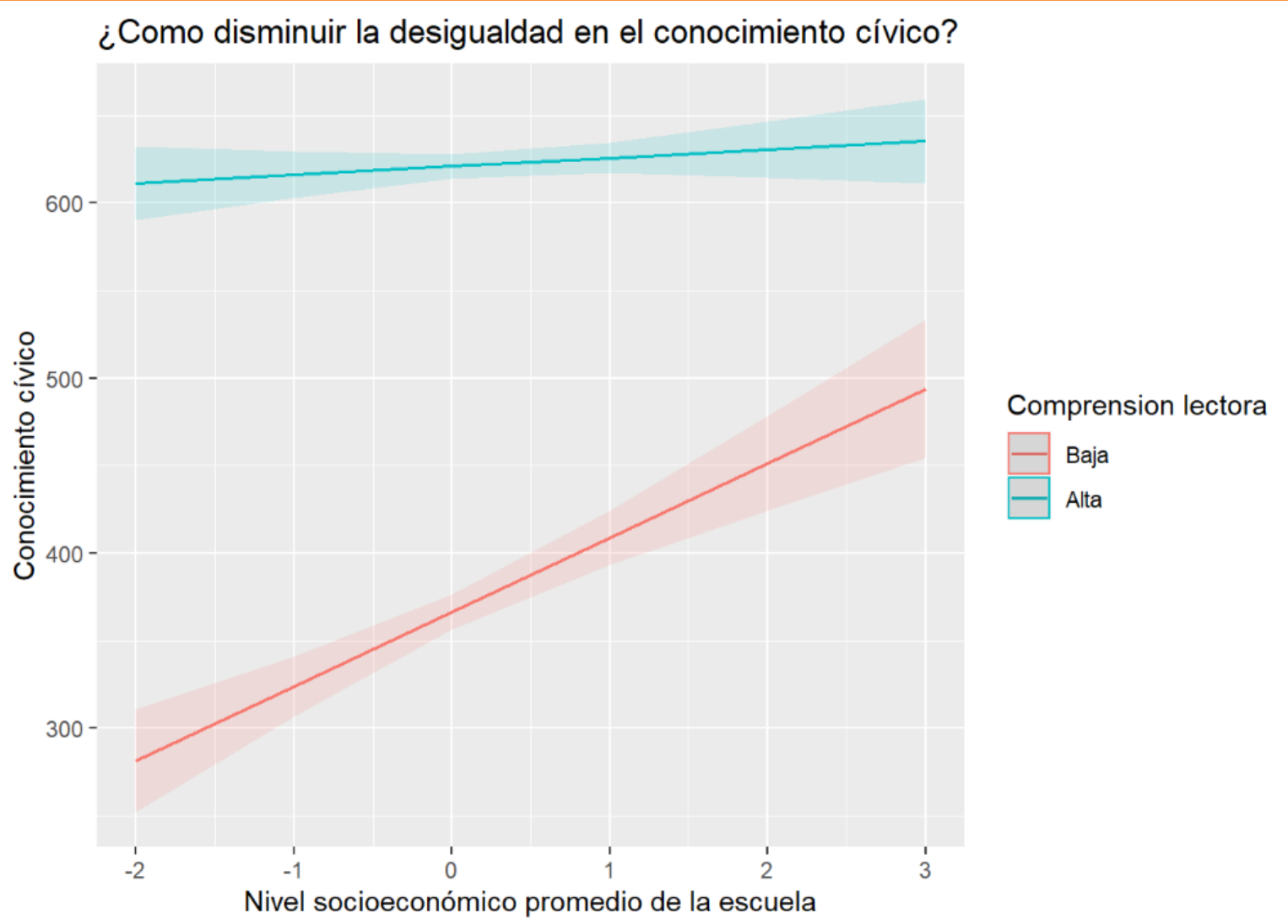

\section{Fuente: Elaboración propia}

En este gráfico podemos evaluar como el efecto del nivel socioeconómico de la escuela es moderado de manera casi perfecta por la comprensión lectora. Como puede verse, si un estudiante posee un excelente puntaje en la prueba de comprensión lectora Simce, independiente del nivel socioeconómico de su escuela, tendrá un muy alto puntaje en conocimiento cívico. Hasta este punto, cabe la duda de si todo este efecto de la comprensión lectora es efectivamente propio, o es más bien un reflejo de algún efecto del colegio. Para evaluar que esto no esté ocurriendo y para evaluar la capacidad explicativa de la desigualdad que posee el lenguaje, realizamos a continuación un análisis de mediación 
multinivel, en el cual se trabajara con variables centradas, despejadas de los efectos contextuales, y variables promedio por escuela.

\section{Resultados de la mediación multinivel.}

A continuación, se presentan los resultados del análisis de mediación multinivel. En estos se evalúa la capacidad del lenguaje de explicar la relación entre NSE y conocimiento cívico, haciendo esta evaluación a nivel uno y nivel dos como recomienda la literatura. Para este fin, se evalúa primero, si el NSE se relaciona con lenguaje, luego si el NSE se relaciona con el conocimiento cívico y, posteriormente, si es que el efecto de NSE sobre conocimiento cívico es controlado por la comprensión lectora, repitiendo este proceso en ambos niveles. En la siguiente tabla el nombre de la columna indica la variable dependiente. 


\section{Tabla n³: Mediación Multinivel}

Mediacion multinivel del lenguaje en la desigualdad social del conocimiento civico

\begin{tabular}{|c|c|c|c|c|c|c|}
\hline & \multicolumn{3}{|c|}{ Nivel 1} & \multicolumn{3}{|c|}{ Nivel 2} \\
\hline & $\begin{array}{c}\text { Lenguaje } \\
\text { (C) }\end{array}$ & $\begin{array}{l}\text { C. Civico } \\
\text { (C) }\end{array}$ & $\begin{array}{l}\text { C. Civico } \\
\text { (C) }\end{array}$ & $\begin{array}{c}\text { Lenguaje } \\
\text { (M) }\end{array}$ & $\begin{array}{c}\text { C. Civico } \\
\text { (M) }\end{array}$ & $\begin{array}{c}\text { C. Civico } \\
\text { (M) }\end{array}$ \\
\hline \multirow[t]{2}{*}{ Intercepto } & -0.00 & -0.00 & -0.00 & $245.18^{* * *}$ & $484.59^{* * *}$ & $359.49^{* * * *}$ \\
\hline & $(0.75)$ & $(1.30)$ & $(1.06)$ & $(0.42)$ & $(0.67)$ & $(5.83)$ \\
\hline \multirow[t]{2}{*}{$\operatorname{NSE}(\mathrm{C})$} & $7.49^{* * * *}$ & $18.84^{* * * *}$ & $11.29^{* * *}$ & & & \\
\hline & $(1.04)$ & $(1.82)$ & $(1.50)$ & & & \\
\hline \multirow[t]{2}{*}{ Lenguaje (C) } & & & $1.01^{* * * *}$ & & & \\
\hline & & & $(0.03)$ & & & \\
\hline \multirow[t]{2}{*}{ NSE (M) } & & & & $24.34^{* * *}$ & $55.86^{* * *}$ & $43.31^{* * *}$ \\
\hline & & & & $(0.49)$ & $(0.78)$ & $(0.88)$ \\
\hline \multirow[t]{2}{*}{ Lenguaje (M) } & & & & & & $0.51^{* * * *}$ \\
\hline & & & & & & $(0.02)$ \\
\hline $\mathrm{AIC}$ & 32359.10 & 35861.40 & 34592.96 & -41806.77 & -41542.49 & -48236.68 \\
\hline $\mathrm{BIC}$ & 32383.31 & 35885.60 & 34623.22 & -41782.56 & -41518.28 & -48206.42 \\
\hline Log Likelihood & -16175.55 & -17926.70 & -17291.48 & 20907.39 & 20775.24 & 24123.34 \\
\hline Num. obs. & 3140 & 3140 & 3140 & 3140 & 3140 & 3140 \\
\hline Num. groups: idschool & 152 & 152 & 152 & 152 & 152 & 152 \\
\hline $\begin{array}{l}\text { Var: idschool } \\
\text { (Intercept) }\end{array}$ & 0.00 & 0.00 & 0.00 & 26.31 & 67.00 & 48.87 \\
\hline Var: Residual & 1745.89 & 5326.25 & 3553.92 & 0.00 & 0.00 & 0.00 \\
\hline
\end{tabular}

Fuente: Elaboración propia

A partir de los resultados de la tabla anterior, es posible concluir que la comprensión lectora posee la capacidad de explicar medianamente la desigualdad en el conocimiento cívico. En primer lugar, se puede afirmar con un $99,9 \%$ de confianza que existe una relación significativa entre NSE y manejo del lenguaje a nivel individual, relación según la cual por cada punto que aumenta el NSE, se aumenta 7 puntos en la prueba de lenguaje. En segundo lugar, existe con el mismo criterio de confianza una relación significativa entre NSE y conocimiento cívico. Al controlar dicha relación por la comprensión lectora, podemos apreciar que, si bien el efecto no deja de ser altamente significativo, este disminuye aproximadamente un 40\%. Esta disminución nos indica que aproximadamente el $40 \%$ de la desigualdad social del conocimiento cívico se explica por desigualdades en el manejo del lenguaje. En torno al nivel dos, se puede observar un fenómeno similar, pero en este caso, 
la mediación del lenguaje corresponde a un 22,5\%, lo que nos indica que dicha proporción del efecto del NSE promedio de la escuela hacia el conocimiento cívico promedio de la escuela, pasa por el promedio de comprensión lectora promedio.

A continuación, se presenta una tabla donde se expone un diagrama de senderos, en el cual se contrasto la capacidad mediadora de la comprensión lectora y el interés político. Para ello, al igual que en la tabla anterior, se utilizaron las variables independientes centradas al promedio del grupo. En el caso del interés político, se utilizó la variable de 4 alternativas centrada al promedio del grupo, lo cual genera una variable que puede ser tratada como continua. Además, se pasaron los puntajes a una escala del 0 al 100 con el objetivo de que los efectos sean más comparables. Cabe destacar que estos análisis se realizaron con una tabla igual que la anterior, pero incluyendo más variables. Por ende, debe recordarse que los valores a nivel escuela corresponden a promedios y los valores a nivel estudiante a variables centradas. 


\section{Diagrama $n^{\circ} 2$ : Mediación multinivel}
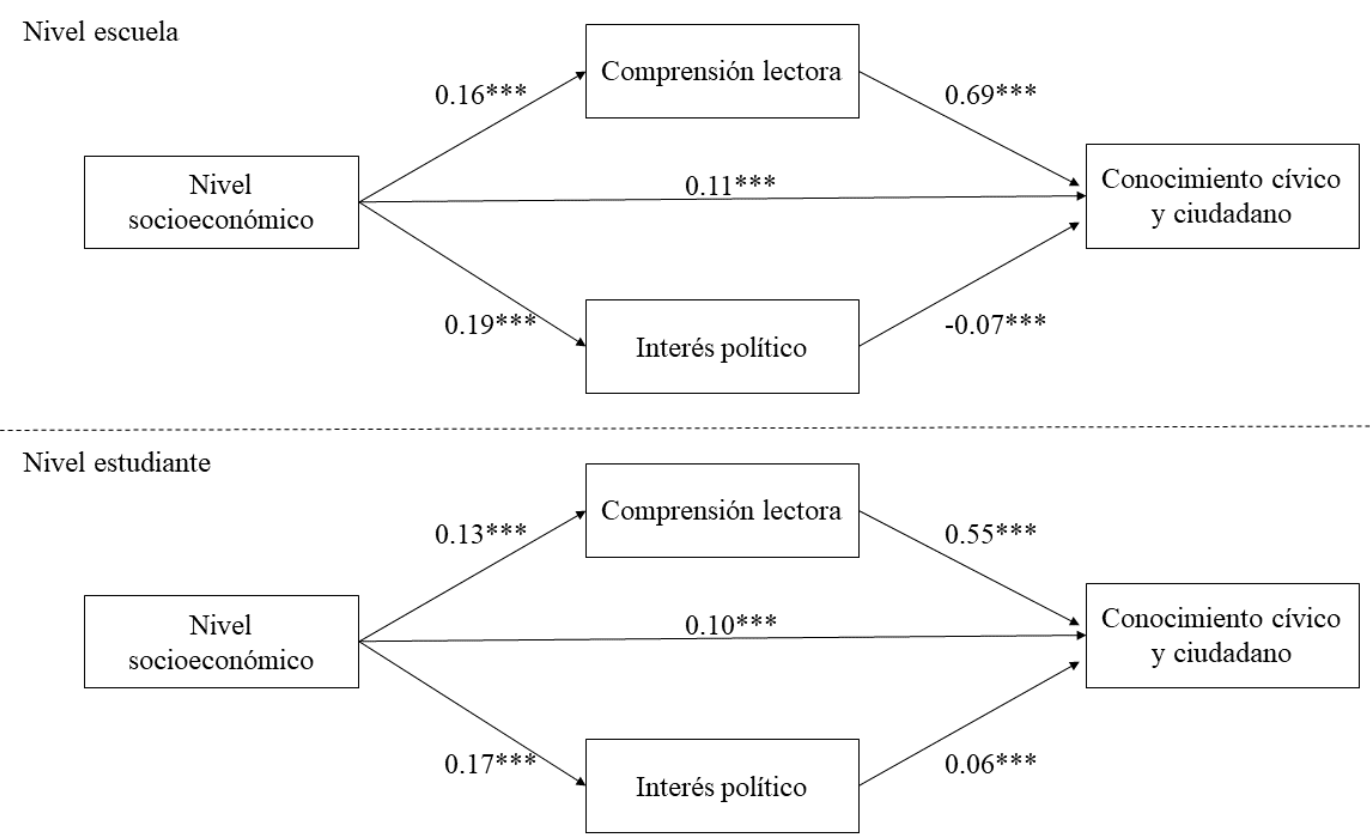

\section{Fuente: Elaboración propia}

A partir del diagrama de referencia y de los resultados del análisis de mediación multinivel, se puede concluir que el manejo del lenguaje explica mucho mejor que el interés político la relación entre NSE y conocimiento cívico. Mientras que el manejo del lenguaje explica un $40 \%$ de la relación el interés en la politica solo explica un 5\% de la misma. Como puede apreciarse en el diagrama, el efecto del NSE sobre el interés político y sobre la comprensión lectora es semejante, no obstante, la relación de estas dos variables y el conocimiento cívico es bastante dispar, siendo el efecto del lenguaje casi 10 veces más grande. 


\section{Conclusiones: respondiendo las incógnitas}

En consideracion de los resultados, podemos entregar algunas respuestas parciales a nuestros objetivos de investigación.

En primer lugar, podemos decir que existe una relación moderadamente alta entre el conocimiento Cívico y la comprensión lectora, relación la cual no solo se mantiene al incluir otras variables fundamentales en el modelo, sino que es capaz de controlar buena parte del efecto de las variables de origen social. La evidencia del efecto de la comprensión lectora no niega el efecto positivo sobre el conocimiento Cívico que pueden tener variables como la cultura democrática del establecimiento, lo cual se evidencia con la mantención de este efecto al controlar por comprensión lectora.

En segundo lugar, en función de los resultados, podemos decir que aquellas teorías que explicaban la relación entre NSE y conocimiento cívico, por la transmisión de valores, si bien son ciertas, poseen una visión parcial de lo que ocurre ya que en buena medida la desigualdad social del conocimiento cívico se relaciona ampliamente con la desigualdad social de la comprensión lectora, la cual se relaciona con el capital cultural objetivado de los padres (más de 100 libros en el hogar) y el acceder a buena educación. Es decir, no es tanto que los padres adinerados eduquen democráticamente a sus hijos, sino que más bien, ya sea por transmisión directa o por acceso a buena educación, les otorgan un mayor manejo del lenguaje, lo cual les permite igualmente incorporar de manera más compleja la realidad política, teniendo más herramientas para comprender, analizar y criticar políticamente.

En tercer lugar, gracias a la evidencia, podemos señalar que la comprensión lectora puede ser una forma de mejorar el conocimiento Cívico en sectores vulnerables, puesto que estudiantes de dichos sectores que si poseen un buen manejo del lenguaje poseen igualmente altas capacidades y conocimientos para la vida ciudadana. En función de lo anterior, se hace necesario estudiar experimentalmente el efecto que podría tener un reforzamiento en lenguaje para la prueba de la ICCS. 


\section{Discusión}

Dados los resultados, y el rol prominente de la comprensión lectora en el conocimiento Cívico, se hace necesario revisar algunas propuestas que intentan explicar el conocimiento Cívico, puesto que pueden caer en resultados espurios al no considerar una variable relevante dentro de sus modelos.

En términos teóricos esta investigación ayuda a profundizar la comprensión de la reproducción social de la desigualdad politica, como Brady et al. (2015) sugerían necesario para avanzar en este campo. Estos resultados evidencias que las diferencias sociales en habilidades políticas, no se deben tanto a la transmisión de valores democráticos, como a la transmisión de habilidades relacionadas con el manejo del lenguaje.

Igualmente, se hace necesario profundizar hasta qué punto la comprensión lectora es necesaria para la vida política o más bien es un problema de error de medida, en el cual se incorpora varianza no deseada a la escala. En consideracion de lo anterior, es posible que, al generar instrumentos con menores niveles de abstracción en la redacción de las situaciones y preguntas, se generen menores diferencias de conocimiento Cívico entre distintos grupos sociales.

Respecto a cuáles son los caminos que deben tomarse para mejorar la desigualdad en el conocimiento cívico y en la participación ciudadana, resulta evidente después de los resultados, que además de reforzar la educación cívica en los colegios como ya se está haciendo (y debe seguirse haciendo, debe fomentarse la comprensión lectora). Incluso podemos decir que cuando se posee altos niveles de comprensión lectora, las diferencias de estatus socioeconómico no afectan de manera sustancial, existiendo estudiantes de colegios con bajo nivel económico que poseen una buena comprensión lectora y cívica. Al respecto, tipos de clases que impliquen lectura colectiva y discusión sobre lo leído, para entender en conjunto el sentido de los textos, puede ser una muy buena estrategia, puesto que está demostrado que los profesores que realizan dichas actividades mejoran el puntaje en lenguaje de sus estudiantes (Lorena Ortega) así como que aulas más participativas poseen un efecto para el conocimiento cívico. Es en dichos espacios de participación donde puede sacarse el provecho al capital cultural de cada estudiante y fomentar el efecto par. 
En relación al nuevo plan de formación ciudadana, y considerando estos resultados es sensato esperar un efecto diferencial de la política en cada establecimiento según el nivel de comprensión lectora del colegio, lo cual está relacionado con el nivel socioeconómico. Quizás sea prudente en miras del objetivo de la política, prestar ayuda especializada a colegios que posean bajos niveles de comprensión lectora. Dentro de los colegios prestar apoyo a los estudiantes con dificultades al respecto también puede ser una buena medida a aplicar en cursos anteriores al último ciclo de tercero cuarto, donde deberán aplicar dichas habilidades en la reflexión ciudadana y en la incorporación de conocimientos cívicos. 


\section{Bibliografía}

ACE. (2017). Estudio internacional de educación cívica y ciudadana, presentación de resultados. Agencia educacion. Retrieved September 17, 2019, from https://www.agenciaeducacion.cl/estudios/estudios-internacionales/iccs/

ACE. (2018). Informe técnico simce 2015. Retrieved from http://archivos.agenciaeducacion.cl/Informe_Tecnico_SIMCE_2015_Final.pdf

AERA. (2011). Report y recommendations for the reauthorization of the institute of education sciences. Washington, D.C: American Educational Research Association.

Aguinis, H., Gottfredson, R. K., y Culpepper, S. A. (2013). Best-practice recommendations for estimating cross-level interaction effects using multilevel modeling. Journal of Management, 39(6), 1490-1528.

https://doi.org/10.1177/0149206313478188

Arendt, H. (2009). Qué es la política? Barcelona; Bs. Aires; México: Paidós; I.C.E. de la Universidad Autónoma de Barcelona.

Arensmeier, C. (2015). Swedish students' conceptual knowledge about civics y citizenship: An interview study. Citizenship Teaching \& Learning, 11(1), 9-27. https://doi.org/10.1386/ctl.11.1.9_1

Aristóteles. (1988). Política (M. García Valdés, Ed.). Madrid: Gredos.

Barahona U, P. (2014). Factores determinantes del rendimiento académico de los estudiantes de la universidad de atacama. Estudios Pedagógicos (Valdivia), 40(1), 2539. https://doi.org/10.4067/S0718-07052014000100002

Baron, R. M., y Kenny, D. A. (1986). The moderator-mediator variable distinction in social psychological research: Conceptual, strategic, y statistical considerations. Journal of Personality y Social Psychology, 51(6), 1173-1182. https://doi.org/10.1037/0022-3514.51.6.1173

Bates, D. (2020). Package "lme4”. Retrieved from https://cran.rproject.org/web/packages/lme4/lme4.pdf

Bernstein, B. (1985). CLASES SOCIALES, LENGUAJE y SOCIALIZACION. Revista Colombiana de Educación, (15). https://doi.org/10.17227/01203916.5117

Bernstein, B. (1988). Poder, educación y conciencia: Sociológia de la transmisión cultural (C. Cox, Ed.). Retrieved from https://repositorio.uahurtado.cl/bitstream/handle/11242/8769/4824.pdf? sequence=1\&isAllo wed $=\mathrm{y}$ 
Boeve-de Pauw, J., y Van Petegem, P. (2010). A cross-national perspective on youth environmental attitudes. The Environmentalist, 30(2), 133-144.

https://doi.org/10.1007/s10669-009-9253-1

Brady, H. E., Schlozman, K. L., y Verba, S. (2015). Political mobility y political reproduction from generation to generation. The ANNALS of the American Academy of Political y Social Science, 657(1), 149-173. https://doi.org/10.1177/0002716214550587

Canales, M. (2006). METODOLOGIAS DE LA INVESTIGACION SOCIAL. Place of publication not identified: LOM EDICIONES.

Caro, D. H., y Schulz, W. (2012). Ten hypotheses about tolerance toward minorities among latin american adolescents. Citizenship, Social y Economics Education, 11(3), 213-234. https://doi.org/10.2304/csee.2012.11.3.213

Castillo, J. C., Miranda, D., Bonhomme, M., Cox, C., y Bascopé, M. (2014). Social inequality y changes in students' expected political participation in chile. Education, Citizenship y Social Justice, 9(2), 140-156. https://doi.org/10.1177/1746197914520650

Coffé, H., y Lago, I. (2020). Explaining gender differences in turnout using panel data across elections. Social Science Quarterly, 101(2), 940-959.

https://doi.org/10.1111/ssqu.12759

Contreras, G., y Navia, P. (2013). DIFERENCIAS GENERACIONALES EN LA PARTICIPACIÓN ELECTORAL EN CHILE, 1988-2010. Revista de Ciencia Política (Santiago), 33(2), 419-441. https://doi.org/10.4067/S0718-090X2013000200001

Desposato, S., y Norrander, B. (2009). The gender gap in latin america: Contextual y individual influences on gender y political participation. British Journal of Political Science, 39(1), 141-162. https://doi.org/10.1017/S0007123408000458

Díaz, O. (2017). Dimensiones y tendencias en la participación política en méxico después de la alternancia (2001-2013). Espacios Públicos, 20(49). Retrieved from https://www.redalyc.org/jatsRepo/676/67656393006/html/index.html

Duarte, R., Escario, J.-J., y Sanagustín, M.-V. (2017). The influence of the family, the school, y the group on the environmental attitudes of european students.

Environmental Education Research, 23(1), 23-42.

https://doi.org/10.1080/13504622.2015.1074660

Durkheim, É. (2010). Educación y sociología. Madrid: Popular.

Duval, D., y Pétry, F. (2018). Citizens' evaluations of campaign pledge fulfillment in canada. Party Politics, 135406881878996. https://doi.org/10.1177/1354068818789968

Echeverría, R. (2011). Ontología del lenguaje. Madrid: J.C. Sáez. 
Evans, M. D. R., Kelley, J., Sikora, J., y Treiman, D. J. (2015). Scholarly culture y occupational success in 31 societies. Comparative Sociology, 14(2), 176-218. https://doi.org/10.1163/15691330-12341345

Ferráns, S. D., y Sandoval-Hernández, A. (2017). The civic competence gaps in chile, colombia y mexico y the factors that account for the civic knowledge gap. In B. GarcíaCabrero, A. Sandoval-Hernández, E. Treviño-Villareal, S. D. Ferráns, y M. G. P. Martínez (Eds.), Civics y citizenship (pp. 155-192). Rotterdam: SensePublishers. https://doi.org/10.1007/978-94-6351-068-4_8

Galston, W. A. (2007). Civic knowledge, civic education, y civic engagement: A summary of recent research. International Journal of Public Administration, 30(6), 623-642. https://doi.org/10.1080/01900690701215888

García, M. (2013). EL LENGUAJE COMO INSTRUMENTO HUMANO BÁSICO. La vida embarazada. Una teoría global sobre la vida terrestre y la evolución. Retrieved from https://www.upv.es/jugaryaprender/vidaembarazada/capitulo1.htm

Gimpel, J. G., Lay, J. C., y Schuknecht, J. E. (2003). Cultivating democracy: Civic environments y political socialization in america. Washington, D.C: Brookings Institution Press.

Herrmann, M. G., y Klaveren, A. van. (2016). Disminución de la participación de la población en organizaciones sociales durante los últimos trece años en chile e implicaciones para la construcción de una política de planificación urbana más participativa. EURE (Santiago), 42(125), 175-203. https://doi.org/10.4067/S025071612016000100008

Hutchings, V. L., y Valentino, N. A. (2004). THE CENTRALITY OF RACE IN AMERICAN POLITICS. Annual Review of Political Science, 7(1), 383-408. https://doi.org/10.1146/annurev.polisci.7.012003.104859

IEA. (2016). International civic y citizenship education study 2016. I. Retrieved from https://www.iea.nl/studies/iea/iccs/2016

Janmaat, J. G. (2013). Civic competences. In M. Print y D. Lange (Eds.), Civic education y competences for engaging citizens in democracies (pp. 51-63). Rotterdam:

SensePublishers. https://doi.org/10.1007/978-94-6209-172-6_5

Joignant, A., Bargsted, M., Somma, N., y Campos, T. (2017). Desigualdades de voz política en chile. https://doi.org/10.13140/RG.2.2.28798.28480

Keer, D. (2015). Ciudadania a nivel nacional, regional e internacional: Una revisión de investigaciones enfoques y debates. In Aprendizaje de la ciudadanía: Contextos, experiencias y resultados. (pp. 111-144). Ediciones Universidad Católica de Chile. Retrieved from https://ediciones.uc.cl/index.php/aprendizaje-de-la-ciudadania-contextosexperiencias-y-resultados-2061.html 
Lara, B., Mizala, A., y Repetto, A. (2010). Una mirada a la efectividad de los profesores en chile. Estudios Públicos, (120). https://doi.org/10.38178/cep.vi120.382

Lechner, N. (1984). La conflictiva y nunca acabada construcción del orden deseado. Santiago, Chile: Facultad Latinoamericana de Ciencias Sociales.

Lijphart, A. (1997). Unequal participation: Democracy's unresolved dilemma presidential address, american political science association, 1996. American Political Science Review, 91(1), 1-14. https://doi.org/10.2307/2952255

Lozano, L., García-Cueto, E., y Gallo, P. (2000). Relación entre motivación y aprendizaje. 12(2). Retrieved from http://www.psicothema.com/pdf/579.pdf

Marqués, I. (2016). Apuntes sobre el informe coleman. Sobre la DifÃcil convivencia de los principios igualitarios en un mundo desigual. International Journal of Sociology of Education, 5(2), 107. https://doi.org/10.17583/rise.2016.2092

Mathieu, J. E., y Taylor, S. R. (2007). A framework for testing meso-mediational relationships in organizational behavior. Journal of Organizational Behavior, 28(2), 141-172. https://doi.org/10.1002/job.436

MINEDUC. (2009). El impacto de la deseabilidad social en los cuestionarios del estudiante ICCS 2009 estudio de validación de los cuestionarios del estudio internacional de educación cívica y formación ciudadana ICCS. Retrieved from https://s3.amazonaws.com/archivos.agenciaeducacion.cl/documentosweb/Estudios+Internacionales/ICCS/ICCS+2009/ICCS_2009_Estudio_validacion_cuestion ario_estudiante.pdf

Miranda, D. (2018). Desigualdad y ciudadanía : Una aproximación intergeneracional (Tesis Doctoral, Pontificia Universidad Católica de Chile). Pontificia Universidad Católica de Chile, Santiago. Retrieved from https://repositorio.uc.cl/handle/11534/22255

Miranda, D., Castillo, J. C., y Cumsille, P. (2018). The political socialization of attitudes toward equal rights from a comparative perspective. In A. Sandoval-Hernández, M. M. Isac, y D. Miranda (Eds.), Teaching tolerance in a globalized world (Vol. 4, pp. 103123). Cham: Springer International Publishing. https://doi.org/10.1007/978-3-31978692-6_7

Miranda, D., Castillo, J. C., y Sandoval-Hernandez, A. (2015). Desigualdad y conocimiento cívico: Chile en comparación internacional. In Aprendizaje de la ciudadanía: Contextos, experiencias y resultados. Retrieved from https://www.researchgate.net/publication/292734236_Desigualdad_y_Conocimiento_Civic o_Chile_en_Comparacion_Internacional

Mondak, J. J. (2020). Citizen grit: Effects of domain-specificity, perseverance, y consistency on political judgment. Personality y Individual Differences, 163, 110059. https://doi.org/10.1016/j.paid.2020.110059 
Mouffe, C. (1999). El retorno de lo político: Comunidad, ciudadanía, pluralismo, democracia radical. Barcelona; Buenos Aires; México: Paidós.

Oxford. (2020). Política. Lexico. Retrieved from

https://www.lexico.com/es/definicion/politica

Palet, A., Aguirre, P. de, y Chile, P. (Eds.). (2017). Desiguales: Orígenes, cambios y desafíos de la brecha social en chile. Santiago, Chile: PNUD : Uqbar Editores.

Park, H. (2008). Home literacy environments y children's reading performance: A comparative study of 25 countries. Educational Research y Evaluation, 14(6), 489-505. https://doi.org/10.1080/13803610802576734

Petričević, V., y Stockemer, D. (2020). Why do citizens not turn out? The effect of election-specific knowledge on turnout in european elections in eastern europe. East European Politics y Societies: y Cultures, 34(3), 591-610. https://doi.org/10.1177/0888325419870228

RAE. (2014). Político, ca. Retrieved from https://dle.rae.es/pol\%C3\%ADtico

Rupp, A. A., y Böhme, K. (2008). Handbook of test development: Steven m. Downing y thomas m. Haladyna, lawrence erlbaum associates, inc., january 2006, 778 pages, US\$89.95 (paperback). International Journal of Testing, 8(1), 111-125. https://doi.org/10.1080/15305050701813433

Schulz, W., Ainley, J., Fraillon, J., Kerr, D., y Losito, B. (2010). ICCS 2009 international report: Civic knowledge, attitudes, y engagement among lower-secondary school students in 38 countries.

Schulz, W., Fraillon, J., Ainley, J., Losito, B., y Keer, D. (2011). Estudio internacional sobre educación cívica y ciudadana MARCO DE LA EVALUACIÓN. Retrieved from https://www.iea.nl/sites/default/files/2019-04/ICCS_2009_Framework_Spanish.pdf

Schulz, W., y IEA. (2010). Initial finds from the IEA international civic y citizenship education study. Amsterdam: IEA.

Schulz, W., Losito, B., Carstens, R., y Fraillon, J. (Eds.). (2016). ICCS 2016 technical report. Retrieved from http://archivos.agenciaeducacion.cl/5_Reporte_tecnico_ICCS_2016.pdf

Tezanos-Pinto, P., Cortés, F., y Concha, M. (2015). Participación política y descontento en chile: Una tensión entre el interés en los temas políticos y la desafección generalizada. In 0719-8493: Vol. 6. Midevidencias (Vols. 1-1-6). Retrieved from https://www.mideuc.cl/wp-content/uploads/2016/MidEvidencias-N6.pdf

Torney, J. (1979). Cross-national political socialization research: The IEA study as an agenda setter for research in latin america. Paper, Paper. 
Torney-Purta, J. (2015). El estudio de la educacion cívica y política: Historia e implicancias para chile y américa latina. In Aprendizaje de la ciudadanía: Contextos experiencias y resultados. (pp. 51-73). Ediciones Universidad Católica de Chile. Retrieved from https://ediciones.uc.cl/index.php/aprendizaje-de-la-ciudadania-contextosexperiencias-y-resultados-2061.html

Treviño, E., Béjares, C., Villalobos, C., y Naranjo, E. (2017). Influence of teachers y schools on students' civic outcomes in latin america. The Journal of Educational Research, 110(6), 604-618. https://doi.org/10.1080/00220671.2016.1164114

Verba, S. (2003). Would the dream of political equality turn out to be a nightmare? Perspectives on Politics, 1(4), 663-679. https://doi.org/10.1017/S1537592703000458

Wasburn, P. C., y Adkins Covert, T. J. (2017). Making citizens. Cham: Springer International Publishing. https://doi.org/10.1007/978-3-319-50243-4

Zhang, T., Torney-Purta, J., y Mislevy, R. J. (2015). Understanding civic cognitive assessment tasks: Associations between linguistic features y students' task performance. Citizenship Teaching \& Learning, 11(1), 29-47.

https://doi.org/10.1386/ctl.11.1.29_1

Zhang, Z., Zyphur, M. J., y Preacher, K. J. (2009). Testing multilevel mediation using hierarchical linear models: Problems y solutions. Organizational Research Methods, 12(4), 695-719. https://doi.org/10.1177/1094428108327450 